\title{
EXISTENCE OF COMPACTLY SUPPORTED SOLUTIONS FOR A DEGENERATE NONLINEAR PARABOLIC EQUATION WITH NONLIPSCHITZ SOURCE TERM*
}

\author{
B. BOUFFANDEAU ${ }^{\dagger}$, D. BRESCH ${ }^{\ddagger}$, B. DESJARDINS ${ }^{\S}$, AND E. GRENIER
}

\begin{abstract}
The aim of this paper is to prove existence of non negative compactly supported solutions for a nonlinear degenerate parabolic equation with a non Lipschitz source term in one space dimension. This equation mimics the properties of the classical $k-\varepsilon$ model in the context of turbulent mixing flows with respect to nonlinearities and support properties of solutions.

To the authors' knowledge, originality of the method relies both in the fact with dealing with a non Lipschitz source term and in the comparison of not only the speed but also the acceleration of the support boundaries.
\end{abstract}

Key words. Degenerate parabolic equation, nonLipschitz source term, k-epsilon model, speed and acceleration of support boundaries.

AMS subject classifications. 35K15, 35K65

1. Introduction. The aim of this note is to study nonlinear degenerate parabolic equations with NonLipschitz source term. We will investigate the properties of non negative solutions of

$$
\begin{aligned}
& \partial_{t} u-\nu \partial_{x}^{2}\left(u^{p}\right)=u^{\alpha}+\psi(u), \\
& u_{\mid t=0}=u_{0} \geq 0,
\end{aligned}
$$

with $x \in \mathbb{R}$ where $\psi \in C^{\infty}$ is an increasing function, satisfying $\psi(0)=0$ and $\psi(x) \leq$ $C x$ for some positive constant $C$ and every $x \geq 0$. We will assume that $p>1$ so that the diffusion is strongly degenerate, and that $\alpha>0$ also satisfies $2-p \leq \alpha<1$, so that the nonlinearity is not Lipschitz.

This equation is a crude simplification of the classical $k-\varepsilon$ system of turbulence for fluids and plasma:

$$
\begin{aligned}
\partial_{t} k+\partial_{x}(k u)+\varepsilon & =\partial_{x}\left(C_{\mu} \frac{k^{2}}{\varepsilon} \partial_{x} k\right)+P \frac{k^{2}}{\varepsilon}, \\
\partial_{t} \varepsilon+\partial_{x}(\varepsilon u)+C_{1} \frac{\varepsilon^{2}}{k} & =\partial_{x}\left(\frac{C_{\mu}}{\sigma_{\varepsilon}} \frac{k^{2}}{\varepsilon} \partial_{x} \varepsilon\right)+C_{2} P k,
\end{aligned}
$$

where $k$ and $\varepsilon$ denote the specific turbulent kinetic energy and its dissipation rate, $u$ the velocity field, and $P$ is proportional to turbulence production terms, due for instance to shear or buoyancy effects. $C_{1}, C_{2}, \sigma_{\varepsilon}, C_{\mu}$ denote positive constants.

More precisely, two difficulties of (2) are studied in the present work: non Lipschitz property of source terms and degeneracy of diffusion. The study is restricted to the

\footnotetext{
${ }^{*}$ Received October 23, 2008; accepted for publication December 30, 2008.

${ }^{\dagger}$ Modélisation Mesure et Applications S.A., 66 av. des Champs Elysées, 75008 Paris, France.

‡UMR 5127 CNRS, Laboratoire de Mathématiques de l'Université de Savoie, Campus Scientifique, 73376 Le Bourget du Lac, France (Didier.Bresch@univ-savoie.fr). The author has been supported by the French Agence Nationale de la Recherche project MathOcean (Grant ANR-08-BLAN-0301-01).

$\S$ Département de Mathématiques et Applications, Ecole Normale Supérieure de Paris, 45 rue d'Ulm, 75230 Paris cedex 05, France.

I Unité de Mathématiques Pures et Appliquées, Ecole Normale Supérieure de Lyon, 46 allée d'Italie, 69364 Lyon cedex 07, France.
} 
scalar case, like for the so called one equation $k-\ell$ turbulence model, where the turbulent length scale $\ell=k^{3 / 2} / \varepsilon$ is taken as a constant:

$$
\partial_{t} k+\partial_{x}(k u)+\frac{k^{3 / 2}}{\ell}=\partial_{x}\left(C_{\mu} k^{1 / 2} \ell \partial_{x} k\right)+P \ell k^{1 / 2} .
$$

This non Lipschitz property of the source term also turns out to be a crucial difficulty in numerical schemes, since spurious perturbations may be dramatically amplified by the positive term in the right hand side " $k^{1 / 2} "$ and destroy the support properties.

Similar nonlinear parabolic equations like (1) appear also in various applications, most frequently to describe phenomena of thermal propagation in an absorptive medium where $u$ denotes the temperature. Note that in these cases, the nonlinear term $u^{\alpha}$ is written in the left-hand side of (1). Such dissipative equations have been studied in different exponent ranges namely for linear diffusion and locally Lipschitz absorption but also for slow or fast diffusion and strong or small absorption. The interested reader is referred to [5], [6], [7] and references cited therein for various coefficients ranges.

The problem of (1) with non Lipschitz nonlinearities is the non-uniqueness of solutions. It is straightforward to prove, for instance, that if $t_{0} \geq 0$,

$$
u(t, x)=\left(t-t_{0}\right)_{+}^{1 /(1-\alpha)}
$$

is a particular solution of (1) which vanishes before $t_{0}$ and which lights up at $t=t_{0}$ (for $k-\varepsilon$ system, this corresponds to a non physical apparition of turbulence in a laminar stable state). In particular, the support of $x \mapsto u(t, x)$ is not compact, even if the initial data is compactly supported and if degenerated viscosities should preserve compact support.

A first idea to avoid the singularity of $u^{\alpha}$ is to define solutions of (1) with initial data $u_{0}^{\varepsilon}=u_{0}+\varepsilon$ and let $\varepsilon$ go 0 . However limits of such solution (which exist since they avoid the singularity of nonlinearity) are not compactly supported, which does not fit our requirements.

The second idea which will be followed here is to regularize $u^{\alpha}$ and to pass into the limit with the regularized nonlinearity, using the maximum principle provided by the scalar structure of (1). The main objective is to keep the support compact. This strongly uses bounds of the velocity and of the acceleration of the support boundaries in order to get a comparison principle. Using this property, the end of the proof that is the compact support property will be based on the existence of self similar solution of the auxiliary equation (10). Note that the method used in [7] is also based on regularization and approximation process linked to the term $u^{\alpha}$. The difference with our study is that, in [7], local analysis is done by means of intersection comparisons with the family of traveling wave solutions of the equation. Remark that complete classification of traveling wave solutions has been performed in [8] in the whole range of exponents.

In our paper, we will prove the following result

THEOREM 1.1. Assume that $u_{0}$ is a smooth non negative function, with a support of the form $[\alpha, \beta]$. Let us assume moreover that the derivatives of $u_{0}^{p}$ at $\alpha$ and $\beta$ do no vanish. Then there exists a smooth, nonnegative, compactly supported solution $u$ to (1) defined for all positive times.

By smooth we mean smooth in the interior of its support. In this paper we will neither investigate the uniqueness of the solution $u$ nor study its regularity near the boundary. 
The proof relies on a careful regularization of $u_{+}^{\alpha}$. We shall say that a sequence of smooth functions $\phi_{\varepsilon}(u)$ is an admissible regularization of $u_{+}^{\alpha}$ provided

H1) $\phi_{\varepsilon}(0)=0$ and $\phi_{\varepsilon}(u)>0$ for every $u>0$.

H2) For $u>0, \phi_{\varepsilon}(u)$ increases as $\varepsilon$ decreases to 0 , and converges to $u_{+}^{\alpha}$.

H3) $\phi_{\varepsilon}$ is derivable in 0 with a positive derivative. Moreover $\phi_{\varepsilon}^{\prime}(u)$ is increasing as $\varepsilon$ decreases and goes to infinity as $\varepsilon$ goes to 0 .

H4) $\phi_{\varepsilon}$ is linear on $[0, \varepsilon]$.

Classical arguments give the existence of global smooth solutions to

$$
\partial_{t} u^{\varepsilon}-\nu \partial_{x}^{2}\left(u_{+}^{\varepsilon}\right)^{p}=\phi_{\varepsilon}\left(u^{\varepsilon}\right)+\psi\left(u^{\varepsilon}\right) .
$$

We therefore have to focus on the convergence of $u_{\varepsilon}$ as $\varepsilon$ goes to 0 . We will use principle maximum combined with estimates on the speed and on the acceleration of the support and with the construction of an explicit super solution (a self similar solution). We think the originality of this work lies in the comparison of not only the speed but also of the acceleration of the support boundaries. Calculation of speed of the support has already been used in various papers dedicated to porous medium equation with strong absorption (namely with $p>1$ and $0<\alpha<1$ ) and we refer the reader to [7] and [5]. Other results have been obtained in [6] related to the case $0<p<\alpha<1$.

2. Speed and acceleration of the support. Let us first study solutions of the nonlinear diffusion equation with a linear source term

$$
\partial_{t} u-\nu \partial_{x}^{2} u^{p}=\lambda u
$$

Let us assume that the support of $u(t,$.$) is of the form ]-\infty, X(t)]$. The main objective of this part is to calculate the speed but also the acceleration of the support of solutions. These expressions will be used, after linearization around zero of the nonlinearity, in last section to establish a comparison principle for solutions with compact support.

For Equations (5) (see for instance [3]), a usual transformation is to define $F(t, x)=p u^{p-1}(t, x)$. Then $F$ is smooth up to the boundary as long as its derivative at the boundary does not vanish. If this derivative does not vanish then $F$ can be factorized in

$$
F(t, x)=p \widetilde{g}(t, X(t)-x)(X(t)-x)_{+}
$$

where $\widetilde{g} \in C^{\infty}\left(R_{+} \times R_{+}^{\star}\right)$. Coming back to $u$, it gives

$$
u(t, x)=f(t, x)(X(t)-x)_{+}^{1 /(p-1)} \quad \text { with } \quad f \in \mathrm{C}^{\infty} \quad \text { and } \quad f=\widetilde{g}^{1 /(p-1)} .
$$

Note that $f$ does not vanish on the boundary if the derivative of $F$ does not. We remark that such transformation has been also used in [7].

Speed of the support. Let $\gamma=1 /(p-1)$. Note that $\gamma=\gamma p-1$. Plugging this expression of $u$ in (5) leads to

$$
\begin{aligned}
& \partial_{t}[f(t, x)](X(t)-x)_{+}^{\gamma}+\gamma f(t, x)(X(t)-x)_{+}^{\gamma-1} \partial_{t} X \\
& -\nu \partial_{x x}\left[f(t, x)^{p}\right](X(t)-x)_{+}^{\gamma p}+2 \gamma \nu p \partial_{x}\left[f(t, x)^{p}\right](X(t)-x)_{+}^{\gamma p-1} \\
& \quad-\nu \gamma p(\gamma p-1) f(t, x)^{p}(X(t)-x)_{+}^{\gamma p-2}=\lambda f(t, x)(X(t)-x)_{+}^{\gamma} .
\end{aligned}
$$


To cancel terms in $(X(t)-x)_{+}^{\gamma-1}$ near the boundary, we must have

$$
V(t)=\partial_{t} X(t)=\nu p(\gamma p-1) f^{p-1}(t, X(t)),
$$

which gives the velocity of the support.

Acceleration of the support. Let us now compute the acceleration of the support $\partial_{t} V(t)$. Dividing $(6)$ by $(X(t)-x)_{+}^{\gamma}$ we get

$$
\partial_{t} f+\frac{\gamma \partial_{t} X f-\nu \gamma p(\gamma p-1) f^{p}}{(X(t)-x)_{+}}-\nu \partial_{x x}\left(f^{p}\right)(X-x)_{+}+2 \gamma \nu p \partial_{x}\left(f^{p}\right)=\lambda f
$$

Note that the second term is not singular since $-\gamma \partial_{t} X f+\nu \gamma p(\gamma p-1) f^{p}=0$ on the boundary, and equals the derivative of this function at $X(t)$. Now

$$
\partial_{t} V(t)=\nu p(\gamma p-1) \partial_{t} f^{p-1}+\nu p(\gamma p-1) V \partial_{x} f^{p-1}
$$

where the derivatives of $f$ are evaluated on the boundary. Let us now express this relation in a simpler way. At first, we write

$$
\nu p(\gamma p-1) \partial_{t} f^{p-1}=\nu p(p-1)(\gamma p-1) f^{p-2} \partial_{t} f
$$

But on the boundary $X(t)$, Relation (8) reads

$$
\partial_{t} f=\lambda f-2 \gamma \nu p^{2} f^{p-1} \partial_{x} f+\gamma V \partial_{x} f-\nu \gamma p^{2}(\gamma p-1) f^{p-1} \partial_{x} f .
$$

Hence

$$
\begin{gathered}
\partial_{t} V=\nu p(\gamma p-1)(p-1) V f^{p-2} \partial_{x} f+\lambda(p-1) V \\
-2 \gamma \nu p^{2}(p-1) f^{p-2} V \partial_{x} f+\gamma V(p-1) V f^{-1} \partial_{x} f-\nu \gamma p^{2}(\gamma p-1) V f^{p-2} V(p-1) \partial_{x} f .
\end{gathered}
$$

Using that $\gamma=p-1$, the terms in $\partial_{x} f$ are given by

$$
(p-1) V^{2} f^{-1}-2 p(p-1) V^{2} f^{-1}+\gamma(1-p)(p-1) V^{2} f^{-1} .
$$

Thus

$$
(p-1)-2 p(p-1)+\gamma(1-p)(p-1)=-2 p(p-1)<0 .
$$

Hence the acceleration of the support is given by

$$
\partial_{t} V(t)=\lambda C_{1} V-C_{2} f^{-1} V^{2} \partial_{x} f .
$$

where $C_{1}=p-1$ and $C_{2}=2 p(p-1)$ are positives constants. The first term describes the acceleration by the source term and the second term the nonlinear amplification or damping (depending on the sign of $\partial_{x} f / f$ ) of the acceleration of front. In particular, as long as $f$ remains bounded in $C_{2}, V$ does not vanish provided initially it does not vanish. 
3. Self-similar solution with compact support. Let us look for explicit selfsimilar solutions to the following model equation with a production term

$$
\partial_{t} u=\partial_{x}^{2} u^{p}+u^{2-p}+u \quad \text { with } 1<p<2 .
$$

These solutions will be chosen as supersolutions in the next section to prove that solutions of (1) has compact support.

Here, we prove the following result

THEOREM 3.1. There exists non zero smooth functions $a(t)$ and $\lambda(t)$, defined for all $t \geq 0$ such that

$$
u(t, x)=a(t)\left(1-\frac{x^{2}}{\lambda^{2}(t)}\right)^{\left(\frac{1}{p-1}\right)}
$$

is a compactly supported, self similar, global particular solution of (10). Moreover, $a(t)$ and $\lambda(t)$ go to $+\infty$ as $t$ goes to $+\infty$.

Proof. Let us define

$$
\xi=\frac{x}{\lambda(t)}
$$

After this change of variable, we get

$$
\lambda(t)^{2} \partial_{t} u-\lambda(t) \lambda(t)^{\prime} \xi \partial_{\xi} u=\partial_{\xi \xi} u^{p}+\lambda(t)^{2} u^{(2-p)}+\lambda(t)^{2} u .
$$

Let $z(t)=\lambda(t)^{2}$. We have

$$
z \partial_{t} u-\frac{1}{2} \xi z^{\prime} \partial_{\xi} u=\partial_{\xi \xi} u^{p}+z u^{(2-p)}+z u .
$$

Using the usual change of variable $u(t, \xi)=w^{1 /(p-1)}(t, \xi)$, the equation reads

$$
z \partial_{t} w-\frac{1}{2} \xi z^{\prime} \partial_{\xi} w=p w \partial_{\xi}^{2} w+\frac{p}{p-1}\left|\partial_{\xi} w\right|^{2}+(p-1)(z+z w) .
$$

Let us now looking for separated variable solutions, that means

$$
w=A(t) s(\xi) \text { where } s(\xi)=1-\xi^{2} .
$$

With such a $w$, identifying powers of $\xi^{2}$, we find the system

$$
\left\{\begin{array}{l}
z A^{\prime}+2 p A^{2}-(p-1) z A-(p-1) z=0 \\
-A z^{\prime}+\frac{4 p A^{2}}{p-1}+(p-1) z=0 .
\end{array}\right.
$$

Summing up the two previous equations, we get

$$
A z^{\prime}-z A^{\prime}-\left(\frac{4 p}{p-1}+2 p\right) A^{2}+(p-1) z A=0,
$$

and dividing by $z A$, it reads

$$
\frac{z^{\prime}}{z}-\frac{A^{\prime}}{A}-\left(\frac{4 p}{p-1}+2 p\right) \frac{A}{z}+(p-1)=0 .
$$


Then we set $f=z / A$ and get a differential equation in $f$

$$
f^{\prime}+(p-1) f-\left(\frac{4 p}{p-1}+2 p\right)=0 .
$$

The solution of the equation is

$$
f(t)=\frac{2 p(p+1)}{(p-1)^{2}}+C e^{t(1-p)}, \quad C \in \mathbb{R} .
$$

Then we replace $z$ by $A f$ in the second equation of System (11)

$$
\begin{gathered}
(A f)^{\prime}-\frac{4 p A}{p-1}-(p-1) f=0 \\
A^{\prime}=-\frac{A f^{\prime}}{f}+\frac{4 p A}{p-1} \frac{1}{f}+(p-1) .
\end{gathered}
$$

Note that $f^{\prime} / f$ goes to 0 and $f$ to a constant as $t$ goes to $+\infty$, therefore, by classical arguments, $A(t)$ exists for all positive times and goes to $+\infty$ as $t$ goes to $+\infty$. Moreover, as $f$ goes to a constant and $A$ to $+\infty, z(t)$ and thus $\lambda(t)$ go to $+\infty$ as $t$ goes to $+\infty$, which ends the proof of the Theorem.

4. Passing to limit (Proof of Theorem 1.1). Let $u^{\varepsilon}$ a sequence of solutions of the regularized equation

$$
\partial_{t} u^{\varepsilon}-\nu \partial_{x}^{2}\left(u_{+}^{\varepsilon}\right)^{p}=\phi_{\varepsilon}\left(u^{\varepsilon}\right)+\psi\left(u^{\varepsilon}\right)
$$

with initial data

$$
u^{\varepsilon}(0, x)=\left(u_{0}(x)-\varepsilon\right)_{+} .
$$

Let us assume that $u_{0}$ has a support of the form ] $-\infty, X_{0}$ ] (the case of supports of the form $\left[X_{0}, Y_{0}\right]$ is similar). Let us assume that $u_{0}$ is smooth in the interior of the domain, and that the first derivative of $u_{0}^{p}$ does not vanish at $X_{0}$. Then it is classical to get existence of solutions $u_{\varepsilon}$ or (12) with supports of the form ] $-\infty, X_{\varepsilon}(t)$ ], smooth in the interior of the domain.

We claim that:

i) $u^{\varepsilon}$ is increasing in $\varepsilon$.

ii) $u^{\varepsilon}$ in bounded.

iii) $u^{\varepsilon}$ converges as $\varepsilon$ tends to 0 , to a compactly supported solution $u$ of Equation (1).

Proof. i) Let $\varepsilon>\eta>0$. Note that $u^{\varepsilon}(0, x)<u^{\eta}(0, x)$ for any $x$ in the support of $u^{\eta}(0,$.$) . Note also that X_{\varepsilon}(0)<X_{\eta}(0)$. Let $S_{\varepsilon}$ be the support of $u^{\varepsilon}$ and $S_{\eta}$ the support of $u^{\eta}$. Out of $S_{\varepsilon} \cup S_{\eta}, u^{\varepsilon}$ and $u^{\eta}$ are both equal 0 .

Let $t_{0}$ be the first time such that either:

(a) there exists $x_{0}$ with $u^{\varepsilon}\left(t_{0}, x_{0}\right)=u^{\eta}\left(t_{0}, x_{0}\right)>0$ (point not at the border)

or

(b) there exists $x_{0}$ with $\left(t_{0}, x_{0}\right) \in \partial S_{\varepsilon} \cap \partial S_{\eta}$. 
Let us investigate the first case (a). At $\left(t_{0}, x_{0}\right)$ we have

$$
\partial_{t}\left(u^{\varepsilon}-u^{\eta}\right)-\nu \partial_{x}^{2}\left(\left(u_{+}^{\varepsilon}\right)^{p}-\left(u_{+}^{\eta}\right)^{p}\right)=\phi_{\varepsilon}\left(u^{\varepsilon}\left(t_{0}, x_{0}\right)\right)-\phi_{\eta}\left(u^{\eta}\left(t_{0}, x_{0}\right)\right) .
$$

As $\phi_{\varepsilon}<\phi_{\eta}$

$$
\phi_{\varepsilon}\left(u^{\varepsilon}\left(t_{0}, x_{0}\right)\right)-\phi_{\eta}\left(u^{\eta}\left(t_{0}, x_{0}\right)\right)<\phi_{\eta}\left(u^{\varepsilon}\left(t_{0}, x_{0}\right)\right)-\phi_{\eta}\left(u^{\eta}\left(t_{0}, x_{0}\right)\right)=0 .
$$

Moreover for all $t \leq t_{0}$ and for all $x$

$$
\left(\left(u_{+}^{\varepsilon}\right)^{p}-\left(u_{+}^{\eta}\right)^{p}\right)(t, x) \leq 0,
$$

and vanishes at $\left(t_{0}, x_{0}\right)$. Hence

$$
\partial_{x}^{2}\left(\left(u_{+}^{\varepsilon}\right)^{p}-\left(u_{+}^{\eta}\right)^{p}\right)\left(t_{0}, x_{0}\right) \leq 0
$$

which leads to

$$
\partial_{t}\left(u^{\varepsilon}-u^{\eta}\right)\left(t_{0}, x_{0}\right)<0
$$

which contradicts the definition of $t_{0}$ and $x_{0}$.

Let us now turn to the second case (b). Note that

$$
X_{\varepsilon}\left(t_{0}\right)=X_{\eta}\left(t_{0}\right)=x_{0} .
$$

Using Section 2 we write solutions $u^{\varepsilon}(t, x)$ under the form

$$
u^{\varepsilon}(t, x)=f_{\varepsilon}(t, x)\left(X_{\varepsilon}(t)-x\right)_{+}^{\gamma} \text { with } f_{\varepsilon} \in \mathrm{C}^{\infty} .
$$

Using (7), we have

$$
X_{\varepsilon}^{\prime}\left(t_{0}\right)=\nu \gamma p f_{\varepsilon}^{p-1}\left(t_{0}, X_{\varepsilon}\left(t_{0}\right)\right)
$$

and

$$
X_{\eta}^{\prime}\left(t_{0}\right)=\nu \gamma p f_{\eta}^{p-1}\left(t_{0}, X_{\eta}\left(t_{0}\right)\right)
$$

because $\phi_{\varepsilon}$ is linear in $u$ near 0 .

Moreover, as $f_{\eta} \geq f_{\varepsilon}$ since $u^{\eta} \geq u^{\varepsilon}$, we have $X_{\varepsilon}^{\prime}\left(t_{0}\right) \geq X_{\eta}^{\prime}\left(t_{0}\right)$. Strict inequality would mean that $X_{\varepsilon}>X_{\eta}$ for $t<t_{0}$ leads to a contradiction. We therefore have to focus on the equality case. For this, we consider the acceleration formula (9). Linearizing $\phi_{\varepsilon}$ around 0, we found for Equation (12) the following speed

$$
X_{\varepsilon}^{\prime \prime}\left(t_{0}\right)=\frac{1}{\gamma} \phi_{\varepsilon}^{\prime}(0) X_{\varepsilon}^{\prime}\left(t_{0}\right)-C_{2} X_{\varepsilon}^{\prime}\left(t_{0}\right)^{2} f_{\varepsilon}^{-1} \partial_{x} f_{\varepsilon}\left(t_{0}, X_{\varepsilon}\left(t_{0}\right)\right) .
$$

Similarly

$$
X_{\eta}^{\prime \prime}\left(t_{0}\right)=\frac{1}{\gamma} \phi_{\eta}^{\prime}(0) X_{\varepsilon}^{\prime}\left(t_{0}\right)-C_{2} X_{\varepsilon}^{\prime}\left(t_{0}\right)^{2} f_{\eta}^{-1} \partial_{x} f_{\eta}\left(t_{0}, X_{\varepsilon}\left(t_{0}\right)\right) .
$$

Moreover, as $\left(f^{\varepsilon}-f^{\eta}\right)\left(t_{0}, x\right) \leq 0$ and reaches a maximum in $x_{0}$ we have $\partial_{x}\left(f^{\varepsilon}-\right.$ $\left.f^{\eta}\right)\left(t_{0}, x_{0}\right) \geq 0$. Then, at $\left(t_{0}, x_{0}\right)$, we have $\partial_{x} f^{\varepsilon} \geq \partial_{x} f^{\eta}$ and since $\phi_{\varepsilon}^{\prime}(0)<\phi_{\eta}^{\prime}(0)(\mathrm{H} 3)$, it implies that

$$
X_{\varepsilon}^{\prime \prime}\left(t_{0}\right)<X_{\eta}^{\prime \prime}\left(t_{0}\right)
$$


Therefore the acceleration of the support of $u_{\eta}$ is larger than the acceleration of the support of $u_{\varepsilon}$. As their speeds are equal at $t_{0}$, this means that the support of $u_{\eta}$ is strictly larger than the support of $u_{\varepsilon}$ on a small interval $] t_{0}, t_{0}+\eta[$, provided $\eta$ is small enough.

Moreover $f^{\varepsilon}\left(t_{0}, x_{0}\right)=f^{\eta}\left(t_{0}, x_{0}\right)$ and for $x<x_{0}, f^{\varepsilon}\left(t_{0}, x\right)<f^{\eta}\left(t_{0}, x\right)$. Moreover as $X_{\varepsilon}^{\prime \prime}\left(t_{0}\right)<X_{\eta}^{\prime \prime}\left(t_{0}\right)$ we have $\partial_{t} f^{\varepsilon}\left(t_{0}, x_{0}\right)<\partial_{t} f^{\eta}\left(t_{0}, x_{0}\right)$. Hence for $t_{0}<t<t_{0}+\eta$ with $\eta$ small enough, for every $x, f^{\varepsilon}\left(t_{0}, x\right)<f^{\eta}(t, x)$. This implies that $\left(t_{0}, x_{0}\right)$ is an isolated zero of $f^{\varepsilon}=f^{\eta}$.

ii) Let $M$ large enough, such that $0 \leq u_{0}(x) \leq M$. Let $\pi(t)$ be the solution of $\partial_{t} \pi=\pi^{\alpha}+C \pi$, with $\pi(0)=M$. Note that $\pi$ is globally defined. Moreover

$$
\partial_{t} \pi-\nu \partial_{x}^{2}\left(\pi_{+}\right)^{p}-\phi_{\varepsilon}(\pi)=\pi^{\alpha}-\phi_{\varepsilon}(\pi)>0
$$

according to Hypothesis (H4). Using (i), $u^{\varepsilon}(t, x) \leq \pi(t)$ for every $t$ and $x$, and $u^{\varepsilon}$ is bounded locally in $t$, uniformly in $\varepsilon$.

iii) Moreover $u^{\varepsilon}(t, x)$ increases as $\varepsilon$ tends to 0 and is bounded, consequently $\forall t, x \in$ $\left(\left[0, t_{0}\right] \times \mathbb{R}\right) \exists u(t, x)$ such that $u^{\varepsilon}(t, x)$ converges to $u(t, x)$. So Theorem of dominated convergence gives that $u^{\varepsilon}$ tends to $u$ in $\mathrm{L}_{\text {loc }}^{1}\left(\mathbb{R}_{t}^{+} \times \mathbb{R}_{x}\right)$ with $|u(t, x)| \leq \pi(t)$. In a similar way $\left(u_{+}^{\varepsilon}\right)^{p}$ tends to $u^{p}$ in $\mathrm{L}_{l o c}^{1}\left(\mathbb{R}_{t}^{+} \times \mathbb{R}_{x}\right)$ with $\left|u_{+}^{p}(t, x)\right| \leq \pi^{p}(t)$ and $\left(u^{\varepsilon}\right)^{\alpha}$ tends to $u^{\alpha}$ in $\mathrm{L}_{\text {loc }}^{1}\left(\mathbb{R}_{t}^{+} \times \mathbb{R}_{x}\right)$ with $\left|u^{\alpha}(t, x)\right| \leq \pi^{\alpha}(t)$. We can then pass the limit in (12) to get that $u$ is solution to (1).

Let us to prove the solution $u$ of equation (1) is compactly supported. Let us assume that the support of $u_{0}(x)$ is strictly embedded in $[-A,+A]$. Let

$$
v(t, x)=a(t)\left(1-\frac{x^{2}}{\lambda^{2}(t)}\right)_{+}^{\frac{1}{p-1}} \quad \text { with } \quad v_{0} \geq u_{0} \geq 0
$$

be a compactly supported solution of equation (10) with $\lambda(0)=A$ and $a(0)>M$. Repeating the arguments of (i) we get $u^{\varepsilon}(t, x)<v(t, x)$ for every $t, x, \varepsilon$ since $\alpha+p \geq 2$. Passing the limit we get $u(t, x) \leq v(t, x)$, which ends the proof.

\section{REFERENCES}

[1] D.G Aronson, L.A. Caffarelli and J.L. Vazquez. Interface with a corner point in onedimensionnal porous media flow, Comm. Pure Applied Maths., 38 (1985), pp. 375-404.

[2] H. BrÉzis and G. Grandall, Uniqueness of solutions of the initial value problem for $u_{t}-\Delta \phi=$ 0, J. Math. Pures et Appl., 58 (1979), pp. 53-163.

[3] P. Daskalopoulos and R. Hamilton, Regularity of the free boundary for the porous medium equation, J. Amer. Math. Soc., 11 (1998), pp. 899-965.

[4] C. Ebmeyer, Regularity in Sobolev spaces for the fast diffusion and the porous medium equation, preprint (2008).

[5] R. FerReira AND J.L. Vazquez, Extinction behavior for fast diffusion equation with absorption, Nonlinear Analysis, 43:8 (2001), pp. 943-985.

[6] R. Ferreira, V. A. Galaktionovb and J. L. Vazquez, Uniqueness of asymptotic profiles for an extinction problem, Nonlinear Analysis, 4:50 (2002), pp. 495-507.

[7] V.A. Galaktionov, S.I. Shmarev and J.L. Vazquez, Regularity of interfaces in diffusion processes under the influence of strong absorption, Arch. Rational Mech. Analysis, 149:3 (1999), pp. 183-212.

[8] M.A. Herrero AND J.L. VAzquez, The one-dimensional nonlinear heat equation wit absorption: regularity of solutions and interfaces, SIAM J. Math. Anal., 18 (1987), pp. 49-167.

[9] A.S. Kalashnikov, The propagation of disturbances in problems of nonlinear heat conduction with absorption, Zh. Vychisl. Mat. i Mat Fiz, 14:4 (1974), pp. 891-905. 
[10] R. Kerner, Degenerated parabolic equations with general non linearities, Nonlinear Analysis TMA, 4:6 (1980), pp. 1043-1062.

[11] S. LASSERRE, Contribution à l'étude mathématique et numérique des solutions à support compact pour des modèles de turbulence compressible, Université Paris 6, 2005. 
B. BOUFFANDEAU ET AL. 